CRYSTALLOGRAPHIC COMMUNICATIONS

ISSN 2056-9890

Received 22 December 2021

Accepted 23 December 2021

Edited by W. T. A. Harrison, University of Aberdeen, Scotland

Keywords: crystal structure; ferrocene; undecane-1,11-diol; hydrogen bonds; $\mathrm{C}-\mathrm{H} \cdots \pi$ contact; Hirshfeld surface analysis.

CCDC reference: 2130725

Supporting information: this article has supporting information at journals.iucr.org/e

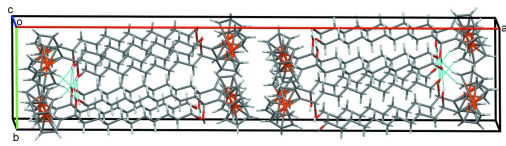

\section{Synthesis, crystal structure and Hirshfeld surface analysis of 1-ferrocenylundecane-1,11-diol}

\author{
C. John McAdam* and Jim Simpson
}

Department of Chemistry, University of Otago, PO Box 56, Dunedin, New Zealand. *Correspondence e-mail: john.mcadam@otago.ac.nz

The racemic title compound, $\left[\mathrm{Fe}\left(\mathrm{C}_{5} \mathrm{H}_{5}\right)\left(\mathrm{C}_{16} \mathrm{H}_{27} \mathrm{O}_{2}\right)\right]$, comprises an $\alpha, \omega$-diolsubstituted undecyl chain with a ferrocenyl substituent at at one terminus. The alkane chain is inclined to the substituted ring of the ferrocene grouping by $84.22(13)^{\circ}$. The ferrocene rings are almost eclipsed and parallel. The crystal structure features $\mathrm{O}-\mathrm{H} \cdots \mathrm{O}$ and $\mathrm{C}-\mathrm{H} \cdots \mathrm{O}$ hydrogen bonds and $\mathrm{C}-\mathrm{H} \cdots \pi$ contacts that stack the molecules along the $c$-axis direction. A Hirshfeld surface analysis reveals that $\mathrm{H} \cdots \mathrm{H}$ interactions $(83.2 \%)$ dominate the surface contacts.

\section{Chemical context}

The title compound, $\mathbf{1}$, is a rare example of a ferrocene molecule substituted with an extended, in this instance 11-membered, alkane chain. It was synthesized to provide a ferrocenyl-substituted diol for the preparation of polyesters with regular pendant electroactive groups. Similar ferrocenyl neo-pentyl diol-derived terephthalate polymers have been shown to display interesting electrochemical properties (McAdam et al., 2008a,b). Friedel-Crafts methodology (Saji et al., 1991) provided the 1-ferrocenyl-undec-10-en-1-one precursor. This was reduced to the racemic alcohol 1-ferrocenyl-undec-10-en-1-ol (2) using $\mathrm{LiAlH}_{4}$. Enantiomeric selection of the individual chiral forms should be possible using more complex synthetic methodology (Ursini et al., 2006; Schwink et al., 1998), but was deemed unnecessary for our purposes. Hydroboration of ferrocenylalkenes has been previously reported (Lo Sterzo et al., 1984) using borane generated in situ from $\mathrm{NaBH}_{4} / \mathrm{BF}_{3} \cdot \mathrm{OEt}_{2}$. Predictably, this method was unsuitable as a means of preparing $\mathbf{1}$ from $\mathbf{2}$, the ferrocenylmethanol moiety being susceptible to attack by $\mathrm{BF}_{3}$, and the resultant loss of $\mathrm{OH}^{-}$abetted by the formation of the stable $\alpha$-ferrocenyl carbenium ion. This prediction was borne out by experiment, the Lewis acid attack resulting in synthesis of 1-ferrocenyl-undec-10-ene and 1-ferrocenyl-undec-11-ol. Instead, a successful synthesis of $\mathbf{1}$ was achieved using hydroboration of $\mathbf{2}$ with 9-BBN.

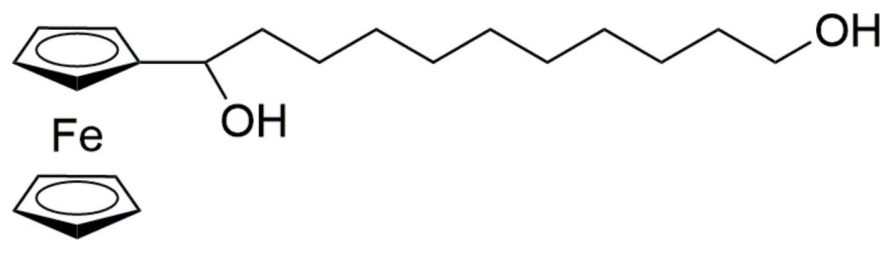




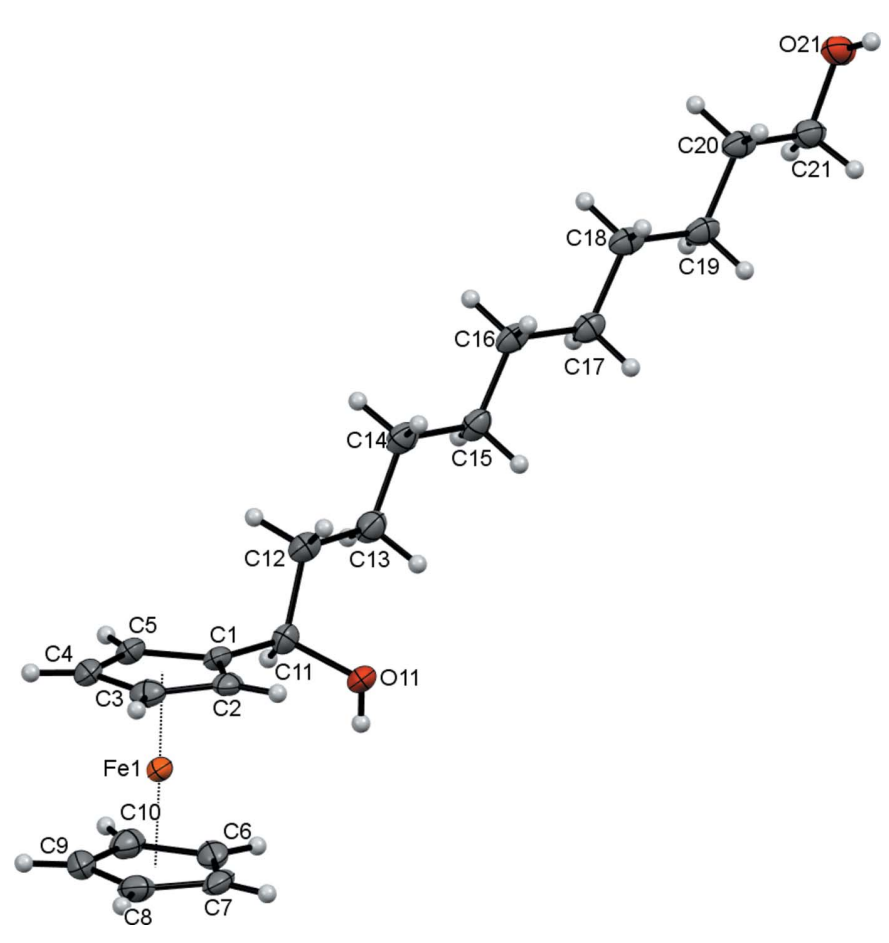

Figure 1

The molecular structure of $\mathbf{1}$ with ellipsoids drawn at the $50 \%$ probability level.

\section{Structural commentary}

The title compound, $\left[\mathrm{Fe}\left(\mathrm{C}_{5} \mathrm{H}_{5}\right)\left(\mathrm{C}_{16} \mathrm{H}_{27} \mathrm{O}_{2}\right)\right]$, comprises a ferrocene unit that carries a well-ordered undecane chain (atoms C11-C21) with hydroxyl substituents at the 1 and 11 positions along the chain (Fig. 1). The $\mathrm{C} 13-\mathrm{C} 12-\mathrm{C} 11-\mathrm{O} 11$ and $\mathrm{C} 19-\mathrm{C} 20-\mathrm{C} 21-\mathrm{O} 21$ torsion angles are 60.9 (3) and $173.9(2)^{\circ}$, respectively. Atom C11 is a stereogenic centre: in the arbitrarily chosen asymmetric molecule it has an $R$ configuration, but crystal symmetry generates a racemic mixture. The alkane chain is almost planar with the r.m.s. deviation from the best fit plane through all $11 \mathrm{C}$ atoms being $0.129 \AA$. This plane is nearly orthogonal to the substituted ferrocene ring with an angle of $84.22(13)^{\circ}$ between them. The $\mathrm{C}_{11}$ undecyl chain in $\mathbf{1}$ is conformationally extended with the typical antiperiplanar (Kane \& Hersh, 2000) arrangement for

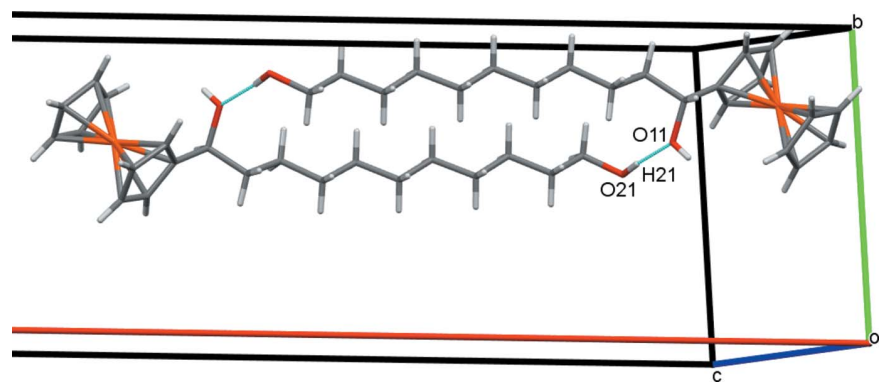

Figure 2

Inversion dimers of $\mathbf{1}$ in the $a b$ plane with $\mathrm{O}-\mathrm{H} \cdots \mathrm{O}$ hydrogen bonds shown as blue lines.

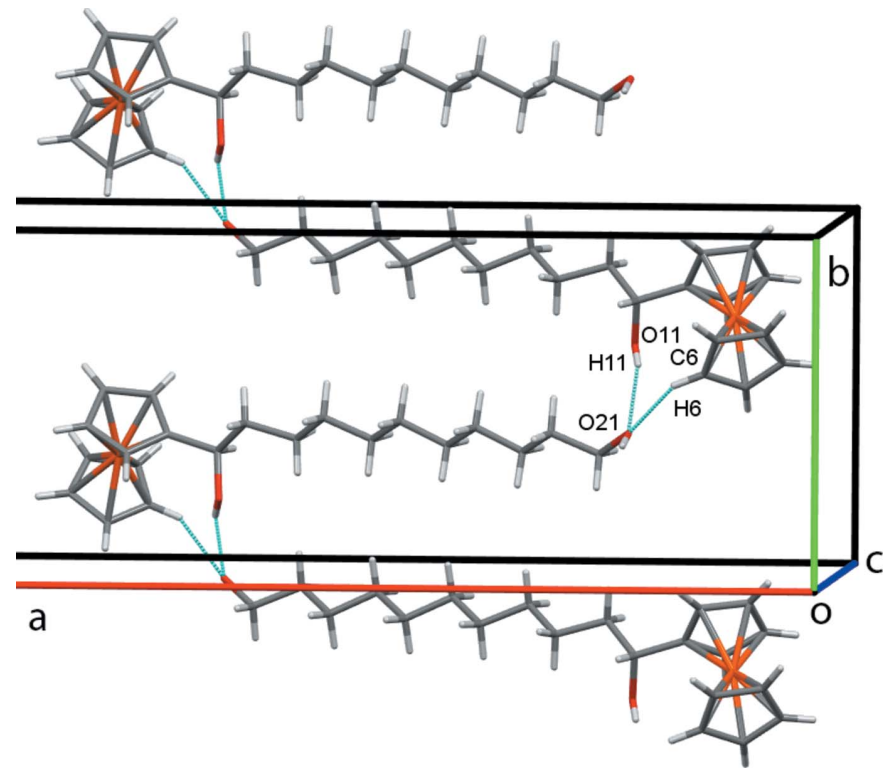

Figure 3

Chains of molecules of $\mathbf{1}$ propagating along $b$ with $\mathrm{O}-\mathrm{H} \cdots \mathrm{O}$ and $\mathrm{C}-$ $\mathrm{H} \cdots \mathrm{O}$ hydrogen bonds shown as blue lines.

$\mathrm{C}_{n}-\mathrm{C}_{n+3}$ groupings and a $\mathrm{C} 11 \cdots \mathrm{C} 21$ separation of 12.627 (4) A. The C1-C5 and C6-C10 cyclopentadienyl rings of the ferrocenyl group are approximately $3^{\circ}$ from being eclipsed and are almost coplanar with a dihedral angle of $1.7(2)^{\circ}$ between them; the separation of the ring centroids is is 3.298 (2) A.

\section{Supramolecular features}

In the crystal of $\mathbf{1}$, inversion dimers form in the $a b$ plane through pairwise classical $\mathrm{O} 21-\mathrm{H} 21 \cdots \mathrm{O} 11$ hydrogen bonds (Table 1 ), which generate $R_{2}^{2}(28)$ ring motifs (Fig. 2). Additional classical $\mathrm{O} 11-\mathrm{H} 11 \cdots \mathrm{O} 21$ hydrogen bonds, supported by weaker non-classical $\mathrm{C} 6-\mathrm{H} 6 \cdots \mathrm{O} 21$ contacts, form alternating chains of molecules along the $b$-axis direction and $\mathrm{O} 21$ acts as a double acceptor (Fig. 3). A weak $\mathrm{C} 7-\mathrm{H} 7 \cdots \mathrm{Cg} 2$ $\left(\mathrm{H} \cdots \pi=2.89 \AA, \mathrm{C}-\mathrm{H} \cdots \pi=164^{\circ}\right)$ contact involving the unsubstituted ring of the ferrocene unit forms double chains of molecules propagating along the $c$-axis direction (Fig. 4)



Figure 4

Double chains of molecules of $\mathbf{1}$ along $c . \mathrm{Cg} 2$ is the centroid of the C6$\mathrm{C} 10$ cyclopentadienyl ring, shown here as red spheres, with the $\mathrm{C}-\mathrm{H} \cdots \pi$ contacts drawn as dashed red lines. 
Table 1

Hydrogen-bond geometry $\left(\AA,^{\circ}\right)$.

\begin{tabular}{lllll}
\hline$D-\mathrm{H} \cdots A$ & $D-\mathrm{H}$ & $\mathrm{H} \cdots A$ & $D \cdots A$ & $D-\mathrm{H} \cdots A$ \\
\hline $\mathrm{O} 11-\mathrm{H} 11 \cdots \mathrm{O} 21^{\mathrm{i}}$ & 0.76 & 2.06 & $2.755(3)$ & 152 \\
$\mathrm{O}^{\mathrm{ii}} 1-\mathrm{H} 21 \cdots \mathrm{O} 11^{\mathrm{ii}}$ & 0.83 & 1.90 & $2.726(3)$ & 175 \\
$\mathrm{C}^{-\mathrm{H} 6} \cdots \mathrm{O} 21^{\mathrm{i}}$ & 0.95 & 2.60 & $3.380(4)$ & 140 \\
\hline
\end{tabular}

Symmetry codes: (i) $-x+\frac{1}{2}, y-\frac{1}{2},-z+\frac{3}{2}$; (ii) $-x+\frac{1}{2},-y+\frac{3}{2},-z+2$.

Table 2

Percentage contributions to the Hirshfeld surface of $\mathbf{1}$.

\begin{tabular}{ll}
\hline Contents & Included surface area \\
\hline $\mathrm{H} \cdots \mathrm{H}$ & 83.2 \\
$\mathrm{H} \cdots \mathrm{C} / \mathrm{C} \cdots \mathrm{H}$ & 9.4 \\
$\mathrm{H} \cdots \mathrm{O} / \mathrm{O} \cdots \mathrm{H}$ & 7.3 \\
\hline
\end{tabular}

where $C g 2$ is the centroid of the C6-C10 cyclopentadienyl ring. Overall these various contacts combine to stack the molecules of $\mathbf{1}$ along the $c$-axis direction in two discrete, parallel and well-separated columns (Fig. 5).

\section{Hirshfeld surface analysis}

Further details of the intermolecular interactions in $\mathbf{1}$ were obtained using Hirshfeld surface analysis (Spackman \& Jayatilaka, 2009) with Hirshfeld surfaces and two-dimensional fingerprint plots generated with Crystal Explorer (Turner et al., 2017). Hirshfeld surfaces for opposite faces of $\mathbf{1}$ are shown in Fig. 6(a) and (b). Bold red areas on the Hirshfeld surfaces correspond to the classical $\mathrm{O}-\mathrm{H} \cdots \mathrm{O}$ hydrogen bonds while the weaker $\mathrm{C}-\mathrm{H} \cdots \mathrm{O}$ and $\mathrm{C}-\mathrm{H} \cdots \pi$ contacts appear as faint red circles. Fingerprint plots (Fig. 7) reveal that $\mathrm{H} \cdots \mathrm{H}$ interactions dominate the surface contacts, as would be expected for a molecule with such a predominance of $\mathrm{H}$ atoms, with $\mathrm{H} \cdots \mathrm{C} / \mathrm{C} \cdots \mathrm{H}$ and $\mathrm{H} \cdots \mathrm{O} / \mathrm{O} \cdots \mathrm{H}$ contacts also making significant contributions to the surface (Table 2).

\section{Database survey}

Ferrocene derivatives with pendant $\mathrm{C}_{n}$ alkyl chains $(n \geq 11)$ are uncommon and the majority of such structures that appear in the Cambridge Structural Database (version 5.41 Nov 2019 with updates to March 2020; Groom et al., 2016) are bisferrocenyl complexes. These include 1,12-bis-ferrocenyldodecane (refcodes FOHHAM and FOHHAM01; Bequeath et al., 2005, Wedeking et al., 2006a) and the tetradecane, octadecane and docosane derivatives (VEFXIO, VEFXOU,

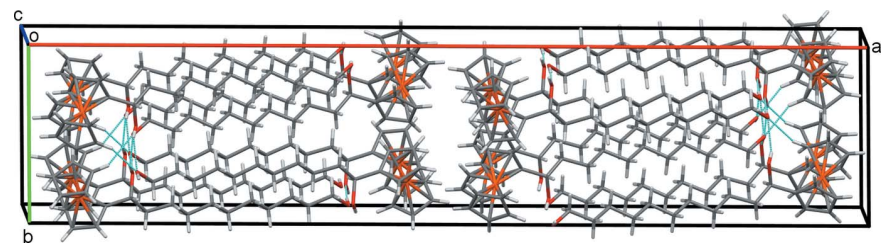

Figure 5

Overall packing of $\mathbf{1}$ viewed along the $c$-axis direction. (a)

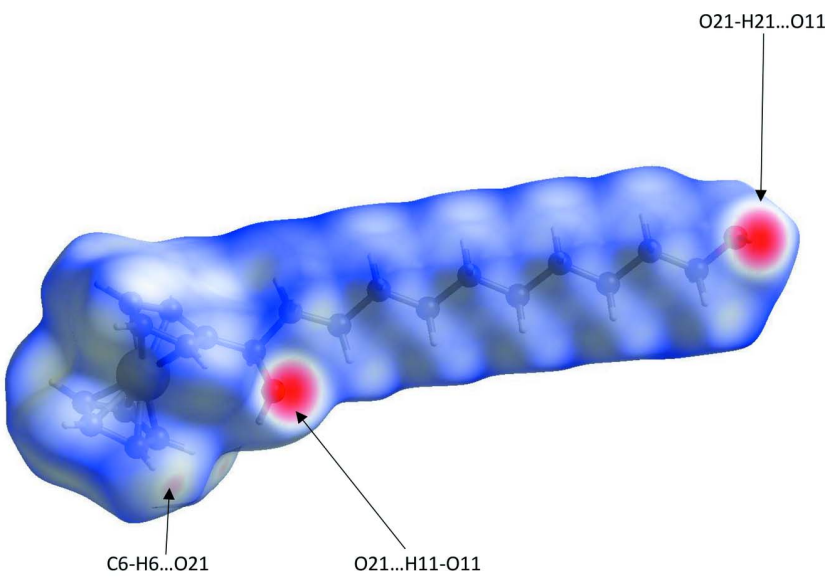

(b)

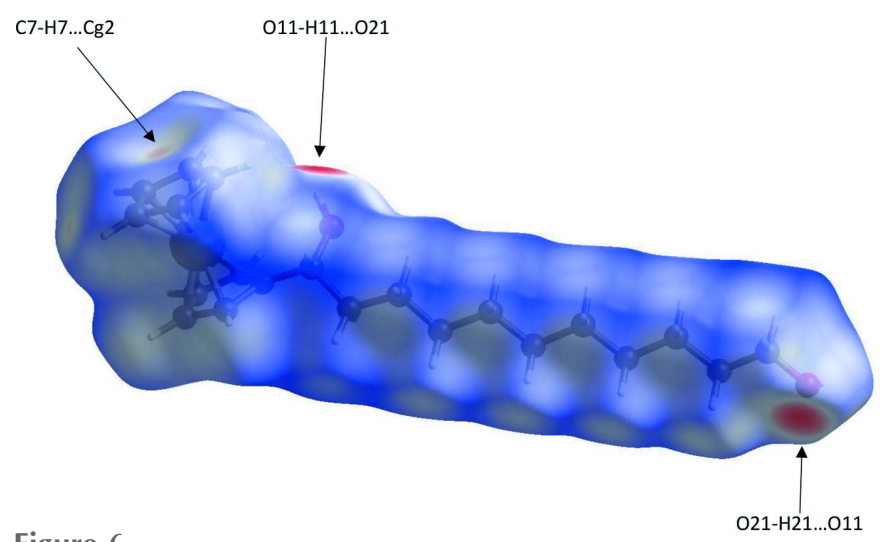

Figure 6

O21-H21...O11

Hirshfeld surfaces for opposite faces of $\mathbf{1}$ mapped over $d_{\text {norm }}$ in the range -0.67 to 1.35 a.u.

VEFXUA; Wedeking et al., 2006a). n-Tetradecylferrocene (MEFRUL; Wedeking et al., 2006b) is the only mono-ferrocene with an unsubstituted alkane chain, while our earlier

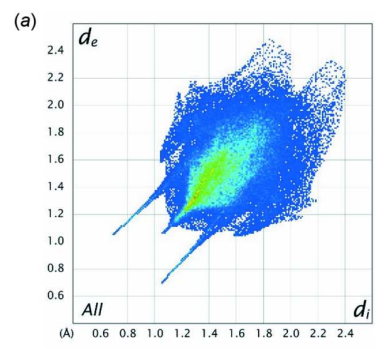

(b)

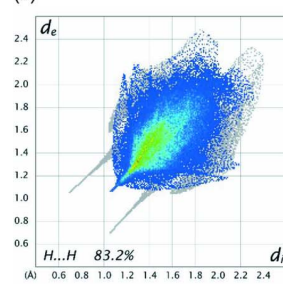

(c)
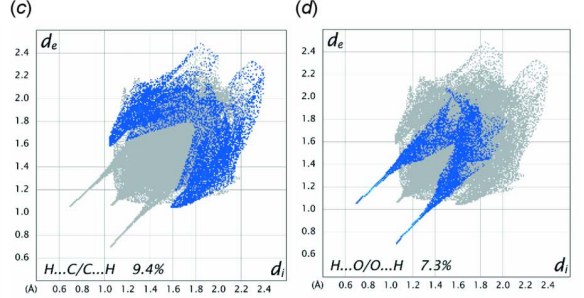

Figure 7

A full two-dimensional fingerprint plot for 1, $(a)$, together with $(b)-(d)$ separate principal contact types for the molecule: $\mathrm{H} \cdots \mathrm{H}, \mathrm{H} \cdots \mathrm{C} / \mathrm{C} \cdot \mathrm{H}$ and $\mathrm{H} \cdots \mathrm{O} / \mathrm{O} \cdots \mathrm{H}$, respectively. 


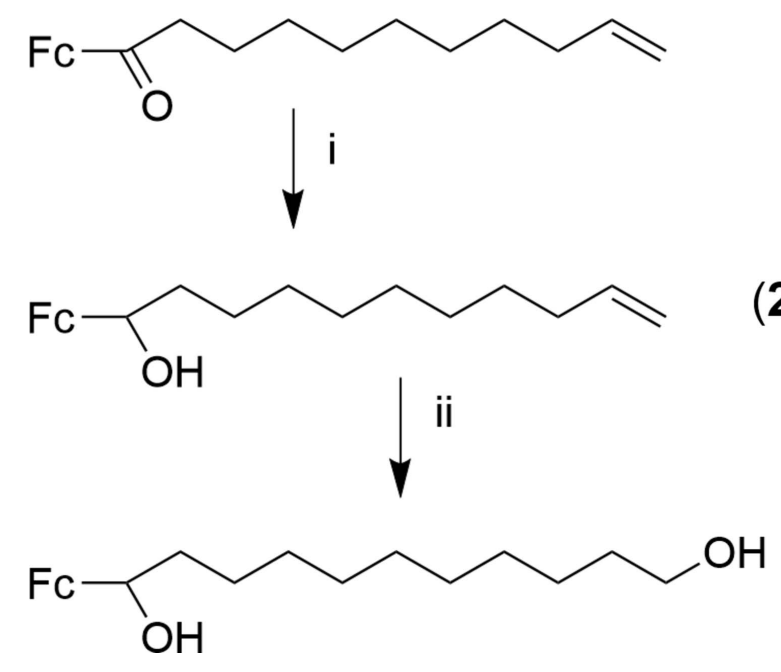

Figure 8

Preparation scheme for $\mathbf{1}$; (i) $\mathrm{LiAlH}_{4}, \mathrm{Et}_{2} \mathrm{O}$; (ii) 9-BBN, THF.

report of the structure of 11-bromo-1-ferrocenylundecan-1one (LICNIV; McAdam et al., 2007) is the sole example of such a structure with substitution on the alkane chain. Interestingly, the structure of the related 1,11-undecanediol (HIYHAY; Nakamura et al., 1999) has also been reported. However, $\alpha, \omega$-dihydroxyalkane $\left(\mathrm{C}_{n}, n \geq 10\right)$ structures are uncommon and often crystallize as co-crystals, see, for example, KEXZOD and KEXZUJ (Loehlin et al., 2007) OTIZEX, OTIZIB, OTIZOH and OTIZUN (Martí-Rujas et al., 2011).

\section{Synthesis and crystallization}

The title compound $\mathbf{1}$ was prepared in two steps from 1-ferrocenyl-undec-10-en-1-one (Evans et al., 2008) via a lithium aluminium hydride reduction followed by hydroboration with 9-borabicyclo[3.3.1]nonane (9-BBN) (Aristoff $e t$ al., 1985), Fig. 8. $\mathrm{LiAlH}_{4}(0.10 \mathrm{~g}, 2.6 \mathrm{mmol})$ was added to 1-ferrocenyl-undec-10-en-1-one $(0.615 \mathrm{~g}, 1.75 \mathrm{mmol})$ in $\mathrm{Et}_{2} \mathrm{O}$ $(10 \mathrm{~mL})$ at $273 \mathrm{~K}$ and stirred for $1 \mathrm{~h}$ before quenching with a few drops of water. The ether fraction was rinsed with saturated $\mathrm{NaCl}$ solution and dried over $\mathrm{MgSO}_{4}$. The solvent was removed under vacuum to give $0.61 \mathrm{~g}(99 \%)$ of the yellow oil 1-ferrocenyl-undec-10-en-1-ol. To this oil, without further purification, in THF $(10 \mathrm{ml})$ was added a solution of 9-BBN (0.5 $M$ in hexane, $3.5 \mathrm{mmol}$ ), the mixture stirred at room temperature for $18 \mathrm{~h}$ before quenching with a few drops of water. The $\mathrm{pH}$ was raised to 8.5 with $\mathrm{NaOH}$, then hydrogen peroxide $\left(30 \%\right.$ in $\left.\mathrm{H}_{2} \mathrm{O}, 7 \mathrm{ml}\right)$ was added and the mixture allowed to stir for another $2 \mathrm{~h}$. The organic layer was rinsed with saturated $\mathrm{NaCl}$ solution and dried over $\mathrm{MgSO}_{4}$. Column chromatography on $\mathrm{SiO}_{2}$ with $\mathrm{CH}_{2} \mathrm{Cl}_{2}$ eluted a trace of the unreacted alcohol. Further elution with EtOAc/ $\mathrm{CH}_{2} \mathrm{Cl}_{2}$ gave the title compound $\mathbf{1}$ as a yellow solid (0.60 g, 94\%). X-ray quality crystals were grown from the mixed solvents of $\mathrm{CH}_{2} \mathrm{Cl}_{2}$ layered with hexane. Analysis calculated for $\mathrm{C}_{21} \mathrm{H}_{32} \mathrm{O}_{2} \mathrm{Fe}$ : C,
Table 3

Experimental details.

\begin{tabular}{|c|c|}
\hline \multicolumn{2}{|l|}{ Crystal data } \\
\hline Chemical formula & {$\left[\mathrm{Fe}\left(\mathrm{C}_{5} \mathrm{H}_{5}\right)\left(\mathrm{C}_{16} \mathrm{H}_{27} \mathrm{O}_{2}\right]\right.$} \\
\hline$M_{\mathrm{r}}$ & 372.31 \\
\hline Crystal system, space group & Monoclinic, $C 2 / c$ \\
\hline Temperature $(\mathrm{K})$ & 92 \\
\hline$a, b, c(\AA)$ & $47.641(3), 10.1522(7), 7.8747(6)$ \\
\hline$\beta\left({ }^{\circ}\right)$ & $97.091(4)$ \\
\hline$V\left(\mathrm{~A}^{3}\right)$ & $3779.6(5)$ \\
\hline$Z$ & 8 \\
\hline Radiation type & Мо $K \alpha$ \\
\hline$\mu\left(\mathrm{mm}^{-1}\right)$ & 0.81 \\
\hline Crystal size $(\mathrm{mm})$ & $0.32 \times 0.14 \times 0.04$ \\
\hline \multicolumn{2}{|l|}{ Data collection } \\
\hline Diffractometer & $\mathrm{CCD}$ area detector \\
\hline Absorption correction & $\begin{array}{l}\text { Multi-scan (SADABS; Bruker, } \\
\text { 2011) }\end{array}$ \\
\hline$T_{\min }, T_{\max }$ & $0.784,1.000$ \\
\hline $\begin{array}{l}\text { No. of measured, independent and } \\
\text { observed }[I>2 \sigma(I)] \text { reflections }\end{array}$ & $16666,2527,2150$ \\
\hline$R_{\text {int }}$ & 0.049 \\
\hline$\theta_{\max }\left({ }^{\circ}\right)$ & 22.7 \\
\hline$(\sin \theta / \lambda)_{\max }\left(\AA^{-1}\right)$ & 0.544 \\
\hline \multicolumn{2}{|l|}{ Refinement } \\
\hline$R\left[F^{2}>2 \sigma\left(F^{2}\right)\right], w R\left(F^{2}\right), S$ & $0.037,0.106,1.06$ \\
\hline No. of reflections & 2527 \\
\hline No. of parameters & 221 \\
\hline $\mathrm{H}$-atom treatment & $\begin{array}{l}\mathrm{H} \text { atoms treated by a mixture of } \\
\text { independent and constrained } \\
\text { refinement }\end{array}$ \\
\hline$\Delta \rho_{\max }, \Delta \rho_{\min }\left(\mathrm{e} \AA^{-3}\right)$ & $0.64,-0.31$ \\
\hline
\end{tabular}

Computer programs: APEX2 and SAINT (Bruker, 2011), SHELXT (Sheldrick, 2015a), SHELXL2018/1 (Sheldrick, 2015b), TITAN (Hunter \& Simpson, 1999), Mercury (Macrae et al., 2020), enCIFer (Allen et al., 2004), PLATON (Spek, 2020), publCIF (Westrip 2010) and Win GX (Farrugia 2012).

67.74; H, 8.66. Found: C, 67.94; H, 8.92\%. ${ }^{1} \mathrm{H}$ NMR $\left(\mathrm{CDCl}_{3}\right)$ : $4.30(m, 1 \mathrm{H},-\mathrm{CHOH}-), 4.24\left(m, 1 \mathrm{H}, \mathrm{C}_{5} H_{4}\right), 4.20(s, 5 \mathrm{H}, \mathrm{Cp})$, $4.17\left(m, 3 \mathrm{H}, \mathrm{C}_{5} \mathrm{H}_{4}\right), 3.64\left(m, 2 \mathrm{H},-\mathrm{CH}_{2}-\mathrm{OH}\right), 1.92[d(\mathrm{~J}=$ $4 \mathrm{~Hz}), 1 \mathrm{H}, \mathrm{Fc}-\mathrm{CHOH}], 1.7-1.3\left[\mathrm{~m}, 18 \mathrm{H},-\left(\mathrm{CH}_{2}\right)_{9}-\right] .{ }^{13} \mathrm{C} \mathrm{NMR}$ $\left(\mathrm{CDCl}_{3}\right)$ : 94.7 (Fc ipso), $69.7(-\mathrm{CHOH}-), 68.3(\mathrm{Cp}), 67.9,67.7$, 67.3, $65.2(\mathrm{Fc}-\mathrm{C} \alpha \& \beta), 63.2\left(-\mathrm{CH}_{2} \mathrm{OH}\right), 38.3,32.9,29.6,29.6$, 29.5, 29.5, 26.1, $25.8\left(-\mathrm{CH}_{2}-\right)$. UV-vis $\left(\mathrm{CH}_{2} \mathrm{Cl}_{2}\right): 325(90), 440$ (110) $\mathrm{nm}(\varepsilon)$.

\section{Refinement}

Crystal data, data collection and structure refinement details are summarized in Table 3. The $\mathrm{O}$-bound $\mathrm{H}$ atoms were located in a difference-Fourier map and their coordinates refined with $\mathrm{U}_{\text {iso }}(\mathrm{H})=1.5 \mathrm{U}_{\text {eq }}(\mathrm{O})$. All $\mathrm{H}$-atoms bound to $\mathrm{C}$ were refined using a riding model with $\mathrm{C}-\mathrm{H}=0.95-1.00 \AA$ and $U_{\text {iso }}(\mathrm{H})=1.2 U_{\text {eq }}(\mathrm{C})$. Despite repeated attempts to grow crystals of better quality, the crystals obtained were weakly diffracting and the extent of diffraction observed is poor with $\sin \left(\theta_{\max }\right) / \lambda=0.544\left(2 \theta_{\max }=44.5^{\circ}\right)$. Despite this, the structure solved and refined adequately.

\section{Acknowledgements}

We thank the New Zealand Ministry of Business, Innovation and Employment Science Investment Fund (grant No. UOO- 
X1206) for support of this work and the University of Otago for the purchase of the diffractometer. JS also thanks the Department of Chemistry, University of Otago for support of his work.

\section{Funding information}

Funding for this research was provided by: Ministry for Business Innovation and Employment (grant No. UOOX1206).

\section{References}

Allen, F. H., Johnson, O., Shields, G. P., Smith, B. R. \& Towler, M. (2004). J. Appl. Cryst. 37, 335-338.

Aristoff, P. A., Johnson, P. D. \& Harrison, A. W. (1985). J. Am. Chem. Soc. 107, 7967-7974.

Bequeath, D. M., Porter, R. L., Lufaso, M. W., Wagner, T. R., Kusnic, R. L., Zeller, M. \& Curtin, L. S. (2005). Acta Cryst. E61, m1070m1072.

Bruker (2011). APEX2, SAINT and SADABS. Bruker AXS Inc., Madison, Wisconsin, USA.

Evans, L. A., Apreutesei, D., Mehl, G. H. \& Wadhawan, J. D. (2008). Electrochem. Commun. 10, 1720-1723.

Farrugia, L. J. (2012). J. Appl. Cryst. 45, 849-854.

Groom, C. R., Bruno, I. J., Lightfoot, M. P. \& Ward, S. C. (2016). Acta Cryst. B72, 171-179.

Hunter, K. A. \& Simpson, J. (1999). TITAN2000. University of Otago, New Zealand.

Kane, S. \& Hersh, W. (2000). J. Chem. Educ. 77, 1366.

Loehlin, J. H. \& Okasako, E. L. N. (2007). Acta Cryst. B63, 132-141.

Lo Sterzo, C. \& Ortaggi, G. (1984). J. Chem. Soc. Perkin Trans. 2, pp. 345-348.
Macrae, C. F., Sovago, I., Cottrell, S. J., Galek, P. T. A., McCabe, P., Pidcock, E., Platings, M., Shields, G. P., Stevens, J. S., Towler, M. \& Wood, P. A. (2020). J. Appl. Cryst. 53, 226-235.

Martí-Rujas, J., Kariuki, B. M., Hughes, C. E., Morte-Ródenas, A., Guo, F., Glavcheva-Laleva, Z., Taştemür, K., Ooi, L., Yeo, L. \& Harris, K. D. M. (2011). New J. Chem. 35, 1515-1521.

McAdam, C. J., Moratti, S. C., Robinson, B. H. \& Simpson, J. (2008a). J. Organomet. Chem. 693, 2715-2722.

McAdam, C. J., Nafady, A., Bond, A. M., Moratti, S. C. \& Simpson, J. (2008b). J. Inorg. Organomet. Polym. 18, 485-490.

McAdam, C. J., Robinson, B. H. \& Simpson, J. (2007). Acta Cryst. E63, m1362.

Nakamura, N., Setodoi, S. \& Ikeya, T. (1999). Acta Cryst. C55, 789 791.

Saji, T., Hoshino, K., Ishii, Y. \& Goto, M. (1991). J. Am. Chem. Soc. 113, 450-456.

Schwink, L., Knochel, P., Eberle, T. \& Okuda, J. (1998). Organometallics, 17, 7-9.

Sheldrick, G. M. (2015a). Acta Cryst. A71, 3-8.

Sheldrick, G. M. (2015b). Acta Cryst. C71, 3-8.

Spackman, M. A. \& Jayatilaka, D. (2009). CrystEngComm, 11, 19-32.

Spek, A. L. (2020). Acta Cryst. E76, 1-11.

Turner, M. J., McKinnon, J. J., Wolff, S. K., Grimwood, D. J., Spackman, P. R., Jayatilaka, D. \& Spackman, M. A. (2017). CrystalExplorer17. University of Western Australia, Nedlands, Western Australia; http://hirshfeldsurface.net.

Ursini, C. V., Mazzeo, F. \& Rodrigues, J. A. R. (2006). Tetrahedron Asymmetry, 17, 3335-3340.

Wedeking, K., Mu, Z., Kehr, G., Sierra, J. C., Lichtenfeld, C. M., Grimme, S., Erker, G., Fröhlich, R., Chi, L., Wang, W., Zhong, D. \& Fuchs, H. (2006a). Chem. Eur. J. 12, 1618-1628.

Wedeking, K., Mu, Z., Kehr, G., Fröhlich, R., Erker, G., Chi, L. \& Fuchs, H. (2006b). Langmuir, 22, 3161-3165.

Westrip, S. P. (2010). J. Appl. Cryst. 43, 920-925. 


\section{supporting information}

Acta Cryst. (2022). E78, 149-153 [https://doi.org/10.1107/S205698902101358X]

Synthesis, crystal structure and Hirshfeld surface analysis of 1ferrocenylundecane-1,11-diol

\section{John McAdam and Jim Simpson}

Computing details

Data collection: APEX2 (Bruker, 2011); cell refinement: APEX2 (Bruker, 2011) and SAINT (Bruker, 2011); data reduction: SAINT (Bruker, 2011); program(s) used to solve structure: SHELXT (Sheldrick, 2015a); program(s) used to refine structure: SHELXL2018/1 (Sheldrick, 2015b) and TITAN (Hunter \& Simpson, 1999); molecular graphics: Mercury (Macrae et al., 2020); software used to prepare material for publication: SHELXL2018/1 (Sheldrick, 2015b), enCIFer (Allen et al., 2004), PLATON (Spek, 2020), publCIF (Westrip 2010) and WinGX (Farrugia 2012).

1-Ferrocenylundecane-1,11-diol

Crystal data

$\left[\mathrm{Fe}\left(\mathrm{C}_{5} \mathrm{H}_{5}\right)\left(\mathrm{C}_{16} \mathrm{H}_{27} \mathrm{O}_{2}\right]\right.$

$M_{r}=372.31$

Monoclinic, $C 2 / c$

$a=47.641(3) \AA$

$b=10.1522(7) \AA$

$c=7.8747(6) \AA$

$\beta=97.091(4)^{\circ}$

$V=3779.6(5) \AA^{3}$

$Z=8$

$F(000)=1600$

$D_{\mathrm{x}}=1.309 \mathrm{Mg} \mathrm{m}^{-3}$

Mo $K \alpha$ radiation, $\lambda=0.71073 \AA$

Cell parameters from 4218 reflections

$\theta=2.4-22.4^{\circ}$

$\mu=0.81 \mathrm{~mm}^{-1}$

$T=92 \mathrm{~K}$

Plate, yellow

$0.32 \times 0.14 \times 0.04 \mathrm{~mm}$

Data collection

CCD area detector diffractometer

Radiation source: sealed tube

Graphite monochromator

phi and $\omega$ scans

Absorption correction: multi-scan

(SADABS; Bruker, 2011)

$T_{\min }=0.784, T_{\max }=1.000$

16666 measured reflections

2527 independent reflections

2150 reflections with $I>2 \sigma(I)$

$R_{\text {int }}=0.049$

$\theta_{\max }=22.7^{\circ}, \theta_{\min }=1.7^{\circ}$

$h=-51 \rightarrow 51$

$k=-11 \rightarrow 11$

$l=-8 \rightarrow 7$

Refinement

Refinement on $F^{2}$

Least-squares matrix: full

$R\left[F^{2}>2 \sigma\left(F^{2}\right)\right]=0.037$

$w R\left(F^{2}\right)=0.106$

$S=1.06$

2527 reflections

221 parameters

0 restraints

Hydrogen site location: inferred from neighbouring sites

$\mathrm{H}$ atoms treated by a mixture of independent and constrained refinement

$w=1 /\left[\sigma^{2}\left(F_{\mathrm{o}}^{2}\right)+(0.0649 P)^{2}+3.6265 P\right]$ where $P=\left(F_{\mathrm{o}}^{2}+2 F_{\mathrm{c}}^{2}\right) / 3$

$(\Delta / \sigma)_{\max }=0.001$

$\Delta \rho_{\max }=0.64$ e $\AA^{-3}$

$\Delta \rho_{\min }=-0.31$ e $\AA^{-3}$ 


\section{Special details}

Geometry. All esds (except the esd in the dihedral angle between two 1.s. planes) are estimated using the full covariance matrix. The cell esds are taken into account individually in the estimation of esds in distances, angles and torsion angles; correlations between esds in cell parameters are only used when they are defined by crystal symmetry. An approximate (isotropic) treatment of cell esds is used for estimating esds involving l.s. planes.

Refinement. A reflection effected by the beamstop and two reflections with Fo $>>>\mathrm{Fc}$ were omitted from the final refinement cycles.

Fractional atomic coordinates and isotropic or equivalent isotropic displacement parameters $\left(\AA^{2}\right)$

\begin{tabular}{|c|c|c|c|c|}
\hline & $x$ & $y$ & $z$ & $U_{\text {iso }} * / U_{\text {eq }}$ \\
\hline $\mathrm{C} 1$ & $0.08497(6)$ & $0.8323(3)$ & $0.3254(4)$ & $0.0242(7)$ \\
\hline $\mathrm{C} 2$ & $0.06289(6)$ & $0.7864(3)$ & $0.4173(4)$ & $0.0249(7)$ \\
\hline $\mathrm{H} 2$ & 0.064664 & 0.719397 & 0.502125 & $0.030^{*}$ \\
\hline $\mathrm{C} 3$ & $0.03794(7)$ & $0.8572(3)$ & $0.3612(4)$ & $0.0281(7)$ \\
\hline H3 & 0.020049 & 0.845762 & 0.400751 & $0.034^{*}$ \\
\hline $\mathrm{C} 4$ & $0.04438(6)$ & 0.9489 (3) & $0.2348(4)$ & $0.0263(7)$ \\
\hline $\mathrm{H} 4$ & 0.031523 & 1.009645 & 0.175642 & $0.032 *$ \\
\hline $\mathrm{C} 5$ & $0.07318(6)$ & $0.9340(3)$ & $0.2122(4)$ & $0.0253(7)$ \\
\hline H5 & 0.083009 & 0.982862 & 0.135301 & $0.030^{*}$ \\
\hline $\mathrm{Fe} 1$ & $0.05212(2)$ & $0.76105(4)$ & $0.16036(5)$ & 0.02107 (19) \\
\hline $\mathrm{C} 6$ & $0.06708(7)$ & $0.6085(3)$ & $0.0268(4)$ & $0.0304(8)$ \\
\hline H6 & 0.085748 & 0.573937 & 0.042214 & $0.036^{*}$ \\
\hline $\mathrm{C} 7$ & $0.04426(7)$ & $0.5650(3)$ & $0.1116(4)$ & $0.0302(8)$ \\
\hline H7 & 0.044913 & 0.496850 & 0.194681 & $0.036^{*}$ \\
\hline $\mathrm{C} 8$ & $0.02022(7)$ & $0.6416(3)$ & $0.0500(4)$ & $0.0331(8)$ \\
\hline H8 & 0.001833 & 0.633157 & 0.083682 & $0.040^{*}$ \\
\hline C9 & $0.02838(7)$ & $0.7320(3)$ & -0.0694 (4) & $0.0336(8)$ \\
\hline H9 & 0.016408 & 0.795724 & -0.129627 & $0.040^{*}$ \\
\hline $\mathrm{C} 10$ & $0.05726(7)$ & $0.7129(3)$ & -0.0855 & $0.0321(8)$ \\
\hline H10 & 0.068119 & 0.760822 & -0.157882 & $0.039^{*}$ \\
\hline C11 & $0.11527(6)$ & $0.7882(3)$ & $0.3455(4)$ & $0.0274(7)$ \\
\hline H11A & 0.122055 & 0.786316 & 0.230314 & $0.033^{*}$ \\
\hline O11 & $0.11807(5)$ & 0.65849 (18) & $0.4193(3)$ & $0.0298(5)$ \\
\hline H11 & $0.1142(7)$ & $0.607(2)$ & $0.350(3)$ & $0.045^{*}$ \\
\hline $\mathrm{C} 12$ & $0.13452(6)$ & $0.8767(3)$ & 0.4624 (4) & $0.0298(7)$ \\
\hline $\mathrm{H} 12 \mathrm{~A}$ & 0.128323 & 0.875560 & 0.577879 & $0.036^{*}$ \\
\hline H12B & 0.132689 & 0.968142 & 0.418890 & $0.036^{*}$ \\
\hline $\mathrm{C} 13$ & $0.16570(6)$ & $0.8354(3)$ & 0.4768 (4) & $0.0313(8)$ \\
\hline H13A & 0.166977 & 0.739640 & 0.500383 & $0.038^{*}$ \\
\hline H13B & 0.172652 & 0.850703 & 0.365045 & $0.038^{*}$ \\
\hline C14 & $0.18500(6)$ & $0.9069(3)$ & $0.6140(4)$ & $0.0293(7)$ \\
\hline H14A & 0.176768 & 0.902415 & 0.723312 & $0.035^{*}$ \\
\hline H14B & 0.186169 & 1.000796 & 0.581914 & $0.035^{*}$ \\
\hline $\mathrm{C} 15$ & $0.21465(6)$ & $0.8487(3)$ & 0.6398 (4) & $0.0286(7)$ \\
\hline H15A & 0.213202 & 0.753762 & 0.665954 & $0.034^{*}$ \\
\hline H15B & 0.222958 & 0.856302 & 0.531020 & $0.034^{*}$ \\
\hline C16 & $0.23466(6)$ & $0.9126(3)$ & $0.7808(4)$ & $0.0283(7)$ \\
\hline
\end{tabular}




$\begin{array}{lllll}\text { H16A } & 0.226058 & 0.908646 & 0.888857 & 0.034^{*} \\ \text { H16B } & 0.236941 & 1.006643 & 0.752146 & 0.034^{*} \\ \text { C17 } & 0.26376(6) & 0.8485(3) & 0.8092(4) & 0.0277(7) \\ \text { H17A } & 0.261428 & 0.754213 & 0.836426 & 0.033^{*} \\ \text { H17B } & 0.272404 & 0.853228 & 0.701314 & 0.033^{*} \\ \text { C18 } & 0.28391(6) & 0.9108(3) & 0.9514(4) & 0.0283(7) \\ \text { H18A } & 0.286265 & 1.005048 & 0.924393 & 0.034^{*} \\ \text { H18B } & 0.275331 & 0.905800 & 1.059485 & 0.034^{*} \\ \text { C19 } & 0.31288(6) & 0.8462(3) & 0.9785(4) & 0.0286(7) \\ \text { H19A } & 0.310578 & 0.752371 & 1.007805 & 0.034^{*} \\ \text { H19B } & 0.321323 & 0.849641 & 0.869788 & 0.034^{*} \\ \text { C20 } & 0.33312(6) & 0.9104(3) & 1.1183(4) & 0.0275(7) \\ \text { H20A } & 0.334116 & 1.005977 & 1.094737 & 0.033^{*} \\ \text { H20B } & 0.325615 & 0.899498 & 1.229180 & 0.033^{*} \\ \text { C21 } & 0.36243(6) & 0.8533(3) & 1.1321(4) & 0.0307(8) \\ \text { H21A } & 0.361643 & 0.759959 & 1.167703 & 0.037^{*} \\ \text { H21B } & 0.369024 & 0.855208 & 1.017765 & 0.037^{*} \\ \text { O21 } & 0.38252(4) & 0.9210(2) & 1.2505(3) & 0.0337(6) \\ \text { H21 } & 0.3814(5) & 0.894(3) & 1.349(4) & 0.051^{*}\end{array}$

Atomic displacement parameters $\left(\AA^{2}\right)$

\begin{tabular}{lllllll}
\hline & $U^{11}$ & $U^{22}$ & $U^{33}$ & $U^{12}$ & $U^{13}$ & $U^{23}$ \\
\hline C1 & $0.0313(17)$ & $0.0143(15)$ & $0.0256(17)$ & $-0.0044(12)$ & $-0.0020(13)$ & $-0.0012(13)$ \\
C2 & $0.0363(18)$ & $0.0187(15)$ & $0.0187(16)$ & $-0.0042(13)$ & $-0.0007(13)$ & $-0.0049(13)$ \\
C3 & $0.0297(17)$ & $0.0256(17)$ & $0.0296(17)$ & $-0.0016(13)$ & $0.0061(14)$ & $-0.0079(14)$ \\
C4 & $0.0295(17)$ & $0.0178(16)$ & $0.0300(17)$ & $0.0025(12)$ & $-0.0024(13)$ & $-0.0033(13)$ \\
C5 & $0.0306(17)$ & $0.0154(15)$ & $0.0290(17)$ & $-0.0028(12)$ & $0.0000(13)$ & $0.0001(13)$ \\
Fe1 & $0.0263(3)$ & $0.0149(3)$ & $0.0213(3)$ & $-0.00056(17)$ & $-0.00010(19)$ & $-0.00066(17)$ \\
C6 & $0.0358(19)$ & $0.0259(17)$ & $0.0280(18)$ & $0.0068(14)$ & $-0.0018(14)$ & $-0.0080(14)$ \\
C7 & $0.048(2)$ & $0.0149(15)$ & $0.0263(17)$ & $-0.0020(14)$ & $0.0010(15)$ & $-0.0038(13)$ \\
C8 & $0.0325(18)$ & $0.0266(17)$ & $0.039(2)$ & $-0.0059(14)$ & $-0.0010(15)$ & $-0.0088(15)$ \\
C9 & $0.042(2)$ & $0.0236(17)$ & $0.0314(19)$ & $0.0036(14)$ & $-0.0105(15)$ & $-0.0024(14)$ \\
C10 & $0.047(2)$ & $0.0248(17)$ & $0.0244(18)$ & $-0.0047(15)$ & $0.0044(15)$ & $-0.0020(14)$ \\
C11 & $0.0305(17)$ & $0.0197(16)$ & $0.0306(18)$ & $-0.0005(13)$ & $-0.0014(14)$ & $0.0052(14)$ \\
O11 & $0.0372(13)$ & $0.0157(11)$ & $0.0339(13)$ & $0.0004(9)$ & $-0.0064(10)$ & $-0.0017(9)$ \\
C12 & $0.0339(18)$ & $0.0202(16)$ & $0.0345(19)$ & $-0.0016(13)$ & $0.0017(14)$ & $-0.0006(14)$ \\
C13 & $0.0307(18)$ & $0.0221(17)$ & $0.041(2)$ & $0.0001(13)$ & $0.0043(15)$ & $0.0014(14)$ \\
C14 & $0.0302(18)$ & $0.0221(16)$ & $0.0361(19)$ & $-0.0037(13)$ & $0.0059(14)$ & $0.0018(14)$ \\
C15 & $0.0317(18)$ & $0.0193(16)$ & $0.0355(19)$ & $-0.0017(13)$ & $0.0063(14)$ & $0.0031(13)$ \\
C16 & $0.0351(18)$ & $0.0187(16)$ & $0.0325(18)$ & $-0.0032(13)$ & $0.0092(14)$ & $0.0017(13)$ \\
C17 & $0.0324(18)$ & $0.0211(16)$ & $0.0304(18)$ & $-0.0026(13)$ & $0.0071(14)$ & $0.0017(13)$ \\
C18 & $0.0337(18)$ & $0.0216(16)$ & $0.0307(18)$ & $-0.0056(13)$ & $0.0078(14)$ & $-0.0019(14)$ \\
C19 & $0.0339(18)$ & $0.0199(16)$ & $0.0326(18)$ & $-0.0067(13)$ & $0.0061(14)$ & $0.0001(13)$ \\
C20 & $0.0356(18)$ & $0.0214(16)$ & $0.0261(17)$ & $-0.0065(13)$ & $0.0060(14)$ & $-0.0011(13)$ \\
C21 & $0.0349(19)$ & $0.0244(17)$ & $0.0321(18)$ & $-0.0067(13)$ & $0.0018(15)$ & $-0.0015(14)$ \\
O21 & $0.0407(13)$ & $0.0284(12)$ & $0.0299(12)$ & $-0.0113(10)$ & $-0.0041(10)$ & $0.0033(10)$ \\
& & & & & & \\
\hline & & & & & &
\end{tabular}


Geometric parameters $\left(\AA,{ }^{\circ}\right)$

\begin{tabular}{|c|c|c|c|}
\hline $\mathrm{C} 1-\mathrm{C} 2$ & $1.427(4)$ & $\mathrm{O} 11-\mathrm{H} 11$ & $0.76(4)$ \\
\hline $\mathrm{C} 1-\mathrm{C} 5$ & $1.432(4)$ & $\mathrm{C} 12-\mathrm{C} 13$ & $1.534(4)$ \\
\hline $\mathrm{C} 1-\mathrm{C} 11$ & $1.501(4)$ & $\mathrm{C} 12-\mathrm{H} 12 \mathrm{~A}$ & 0.9900 \\
\hline $\mathrm{C} 1-\mathrm{Fe} 1$ & $2.040(3)$ & $\mathrm{C} 12-\mathrm{H} 12 \mathrm{~B}$ & 0.9900 \\
\hline $\mathrm{C} 2-\mathrm{C} 3$ & $1.413(4)$ & $\mathrm{C} 13-\mathrm{C} 14$ & $1.515(4)$ \\
\hline $\mathrm{C} 2-\mathrm{Fe} 1$ & $2.041(3)$ & $\mathrm{C} 13-\mathrm{H} 13 \mathrm{~A}$ & 0.9900 \\
\hline $\mathrm{C} 2-\mathrm{H} 2$ & 0.9500 & $\mathrm{C} 13-\mathrm{H} 13 \mathrm{~B}$ & 0.9900 \\
\hline $\mathrm{C} 3-\mathrm{C} 4$ & $1.423(4)$ & $\mathrm{C} 14-\mathrm{C} 15$ & $1.521(4)$ \\
\hline $\mathrm{C} 3-\mathrm{Fe} 1$ & $2.043(3)$ & $\mathrm{C} 14-\mathrm{H} 14 \mathrm{~A}$ & 0.9900 \\
\hline $\mathrm{C} 3-\mathrm{H} 3$ & 0.9500 & C14-H14B & 0.9900 \\
\hline $\mathrm{C} 4-\mathrm{C} 5$ & $1.413(4)$ & $\mathrm{C} 15-\mathrm{C} 16$ & $1.517(4)$ \\
\hline $\mathrm{C} 4-\mathrm{Fe} 1$ & $2.042(3)$ & C15-H15A & 0.9900 \\
\hline $\mathrm{C} 4-\mathrm{H} 4$ & 0.9500 & C15-H15B & 0.9900 \\
\hline $\mathrm{C} 5-\mathrm{Fe} 1$ & $2.038(3)$ & $\mathrm{C} 16-\mathrm{C} 17$ & $1.523(4)$ \\
\hline $\mathrm{C} 5-\mathrm{H} 5$ & 0.9500 & $\mathrm{C} 16-\mathrm{H} 16 \mathrm{~A}$ & 0.9900 \\
\hline $\mathrm{Fe} 1-\mathrm{C} 9$ & $2.033(3)$ & C16-H16B & 0.9900 \\
\hline $\mathrm{Fe} 1-\mathrm{C} 10$ & $2.041(3)$ & $\mathrm{C} 17-\mathrm{C} 18$ & $1.520(4)$ \\
\hline $\mathrm{Fe} 1-\mathrm{C} 6$ & $2.049(3)$ & C17-H17A & 0.9900 \\
\hline $\mathrm{Fe} 1-\mathrm{C} 8$ & $2.052(3)$ & C17-H17B & 0.9900 \\
\hline $\mathrm{Fe} 1-\mathrm{C} 7$ & $2.053(3)$ & $\mathrm{C} 18-\mathrm{C} 19$ & $1.519(4)$ \\
\hline $\mathrm{C} 6-\mathrm{C} 7$ & $1.415(4)$ & $\mathrm{C} 18-\mathrm{H} 18 \mathrm{~A}$ & 0.9900 \\
\hline $\mathrm{C} 6-\mathrm{C} 10$ & $1.422(4)$ & C18-H18B & 0.9900 \\
\hline C6- $-\mathrm{H} 6$ & 0.9500 & $\mathrm{C} 19-\mathrm{C} 20$ & $1.518(4)$ \\
\hline $\mathrm{C} 7-\mathrm{C} 8$ & $1.420(4)$ & C19-H19A & 0.9900 \\
\hline $\mathrm{C} 7-\mathrm{H} 7$ & 0.9500 & C19-H19B & 0.9900 \\
\hline $\mathrm{C} 8-\mathrm{C} 9$ & $1.402(5)$ & $\mathrm{C} 20-\mathrm{C} 21$ & $1.504(4)$ \\
\hline $\mathrm{C} 8-\mathrm{H} 8$ & 0.9500 & $\mathrm{C} 20-\mathrm{H} 20 \mathrm{~A}$ & 0.9900 \\
\hline $\mathrm{C} 9-\mathrm{C} 10$ & $1.410(5)$ & $\mathrm{C} 20-\mathrm{H} 20 \mathrm{~B}$ & 0.9900 \\
\hline C9- & 0.9500 & $\mathrm{C} 21-\mathrm{O} 21$ & $1.427(3)$ \\
\hline $\mathrm{C} 10-\mathrm{H} 10$ & 0.9500 & $\mathrm{C} 21-\mathrm{H} 21 \mathrm{~A}$ & 0.9900 \\
\hline $\mathrm{C} 11-\mathrm{O} 11$ & $1.439(3)$ & $\mathrm{C} 21-\mathrm{H} 21 \mathrm{~B}$ & 0.9900 \\
\hline $\mathrm{C} 11-\mathrm{C} 12$ & $1.512(4)$ & $\mathrm{O} 21-\mathrm{H} 21$ & $0.83(4)$ \\
\hline $\mathrm{C} 11-\mathrm{H} 11 \mathrm{~A}$ & 1.0000 & & \\
\hline $\mathrm{C} 2-\mathrm{C} 1-\mathrm{C} 5$ & $107.1(3)$ & $\mathrm{C} 9-\mathrm{C} 8-\mathrm{H} 8$ & 126.0 \\
\hline $\mathrm{C} 2-\mathrm{C} 1-\mathrm{C} 11$ & $127.5(3)$ & $\mathrm{C} 7-\mathrm{C} 8-\mathrm{H} 8$ & 126.0 \\
\hline $\mathrm{C} 5-\mathrm{C} 1-\mathrm{C} 11$ & $125.4(3)$ & $\mathrm{Fe} 1-\mathrm{C} 8-\mathrm{H} 8$ & 126.5 \\
\hline $\mathrm{C} 2-\mathrm{C} 1-\mathrm{Fe} 1$ & $69.60(16)$ & $\mathrm{C} 8-\mathrm{C} 9-\mathrm{C} 10$ & $108.9(3)$ \\
\hline $\mathrm{C} 5-\mathrm{C} 1-\mathrm{Fe} 1$ & $69.37(16)$ & $\mathrm{C} 8-\mathrm{C} 9-\mathrm{Fe} 1$ & $70.63(18)$ \\
\hline $\mathrm{C} 11-\mathrm{C} 1-\mathrm{Fe} 1$ & $127.9(2)$ & $\mathrm{C} 10-\mathrm{C} 9-\mathrm{Fe} 1$ & $70.03(18)$ \\
\hline $\mathrm{C} 3-\mathrm{C} 2-\mathrm{C} 1$ & $108.7(3)$ & $\mathrm{C} 8-\mathrm{C} 9-\mathrm{H} 9$ & 125.5 \\
\hline $\mathrm{C} 3-\mathrm{C} 2-\mathrm{Fe} 1$ & $69.85(17)$ & $\mathrm{C} 10-\mathrm{C} 9-\mathrm{H} 9$ & 125.5 \\
\hline $\mathrm{C} 1-\mathrm{C} 2-\mathrm{Fe} 1$ & $69.47(16)$ & $\mathrm{Fe} 1-\mathrm{C} 9-\mathrm{H} 9$ & 125.4 \\
\hline $\mathrm{C} 3-\mathrm{C} 2-\mathrm{H} 2$ & 125.7 & $\mathrm{C} 9-\mathrm{C} 10-\mathrm{C} 6$ & $107.2(3)$ \\
\hline $\mathrm{C} 1-\mathrm{C} 2-\mathrm{H} 2$ & 125.7 & $\mathrm{C} 9-\mathrm{C} 10-\mathrm{Fe} 1$ & $69.47(18)$ \\
\hline $\mathrm{Fe} 1-\mathrm{C} 2-\mathrm{H} 2$ & 126.6 & $\mathrm{C} 6-\mathrm{C} 10-\mathrm{Fe} 1$ & $69.96(17)$ \\
\hline
\end{tabular}




\begin{tabular}{|c|c|c|c|}
\hline $\mathrm{C} 2-\mathrm{C} 3-\mathrm{C} 4$ & 107.8 & $\mathrm{C} 9-\mathrm{C} 10-\mathrm{H} 10$ & 126.4 \\
\hline $\mathrm{C} 2-\mathrm{C} 3-\mathrm{Fe} 1$ & $69.69(17)$ & $\mathrm{C} 6-\mathrm{C} 10-\mathrm{H} 10$ & 126.4 \\
\hline $\mathrm{C} 4-\mathrm{C} 3-\mathrm{Fe} 1$ & $69.54(16)$ & $\mathrm{Fe} 1-\mathrm{C} 10-\mathrm{H} 10$ & 125.8 \\
\hline $\mathrm{C} 2-\mathrm{C} 3-\mathrm{H} 3$ & 126.1 & $\mathrm{O} 11-\mathrm{C} 11-\mathrm{C} 1$ & $110.8(2)$ \\
\hline $\mathrm{C} 4-\mathrm{C} 3-\mathrm{H} 3$ & 126.1 & $\mathrm{O} 11-\mathrm{C} 11-\mathrm{C} 12$ & $106.2(2)$ \\
\hline $\mathrm{Fe} 1-\mathrm{C} 3-\mathrm{H} 3$ & 126.2 & $\mathrm{C} 1-\mathrm{C} 11-\mathrm{C} 12$ & $113.0(2)$ \\
\hline $\mathrm{C} 5-\mathrm{C} 4-\mathrm{C} 3$ & $108.3(3)$ & $\mathrm{O} 11-\mathrm{C} 11-\mathrm{C} 21$ & $79.36(14)$ \\
\hline $\mathrm{C} 5-\mathrm{C} 4-\mathrm{Fe} 1$ & $69.60(16)$ & $\mathrm{C} 1-\mathrm{C} 11-\mathrm{C} 21$ & $149.62(17)$ \\
\hline $\mathrm{C} 3-\mathrm{C} 4-\mathrm{Fe} 1$ & $69.67(16)$ & $\mathrm{O} 11-\mathrm{C} 11-\mathrm{H} 11 \mathrm{~A}$ & 108.9 \\
\hline $\mathrm{C} 5-\mathrm{C} 4-\mathrm{H} 4$ & 125.8 & $\mathrm{C} 1-\mathrm{C} 11-\mathrm{H} 11 \mathrm{~A}$ & 108.9 \\
\hline $\mathrm{C} 3-\mathrm{C} 4-\mathrm{H} 4$ & 125.8 & $\mathrm{C} 12-\mathrm{C} 11-\mathrm{H} 11 \mathrm{~A}$ & 108.9 \\
\hline $\mathrm{Fe} 1-\mathrm{C} 4-\mathrm{H} 4$ & 126.5 & $\mathrm{C} 21-\mathrm{C} 11-\mathrm{H} 11 \mathrm{~A}$ & 93.4 \\
\hline $\mathrm{C} 4-\mathrm{C} 5-\mathrm{C} 1$ & $108.1(3)$ & $\mathrm{C} 11-\mathrm{O} 11-\mathrm{H} 11$ & 109.5 \\
\hline $\mathrm{C} 4-\mathrm{C} 5-\mathrm{Fe} 1$ & $69.87(16)$ & $\mathrm{C} 11-\mathrm{C} 12-\mathrm{C} 13$ & $113.0(3)$ \\
\hline $\mathrm{C} 1-\mathrm{C} 5-\mathrm{Fe} 1$ & $69.49(16)$ & $\mathrm{C} 11-\mathrm{C} 12-\mathrm{H} 12 \mathrm{~A}$ & 109.0 \\
\hline $\mathrm{C} 4-\mathrm{C} 5-\mathrm{H} 5$ & 125.9 & $\mathrm{C} 13-\mathrm{C} 12-\mathrm{H} 12 \mathrm{~A}$ & 109.0 \\
\hline $\mathrm{C} 1-\mathrm{C} 5-\mathrm{H} 5$ & 125.9 & $\mathrm{C} 11-\mathrm{C} 12-\mathrm{H} 12 \mathrm{~B}$ & 109.0 \\
\hline $\mathrm{Fe} 1-\mathrm{C} 5-\mathrm{H} 5$ & 126.3 & $\mathrm{C} 13-\mathrm{C} 12-\mathrm{H} 12 \mathrm{~B}$ & 109.0 \\
\hline $\mathrm{C} 9-\mathrm{Fe} 1-\mathrm{C} 5$ & $120.61(12)$ & $\mathrm{H} 12 \mathrm{~A}-\mathrm{C} 12-\mathrm{H} 12 \mathrm{~B}$ & 107.8 \\
\hline $\mathrm{C} 9-\mathrm{Fe} 1-\mathrm{C} 1$ & $156.53(13)$ & $\mathrm{C} 14-\mathrm{C} 13-\mathrm{C} 12$ & $114.8(3)$ \\
\hline $\mathrm{C} 5-\mathrm{Fe} 1-\mathrm{C} 1$ & $41.13(11)$ & $\mathrm{C} 14-\mathrm{C} 13-\mathrm{H} 13 \mathrm{~A}$ & 108.6 \\
\hline $\mathrm{C} 9-\mathrm{Fe} 1-\mathrm{C} 10$ & $40.51(13)$ & $\mathrm{C} 12-\mathrm{C} 13-\mathrm{H} 13 \mathrm{~A}$ & 108.6 \\
\hline $\mathrm{C} 5-\mathrm{Fe} 1-\mathrm{C} 10$ & $106.43(12)$ & $\mathrm{C} 14-\mathrm{C} 13-\mathrm{H} 13 \mathrm{~B}$ & 108.6 \\
\hline $\mathrm{C} 1-\mathrm{Fe} 1-\mathrm{C} 10$ & $121.14(13)$ & $\mathrm{C} 12-\mathrm{C} 13-\mathrm{H} 13 \mathrm{~B}$ & 108.6 \\
\hline $\mathrm{C} 9-\mathrm{Fe} 1-\mathrm{C} 2$ & $160.79(14)$ & $\mathrm{H} 13 \mathrm{~A}-\mathrm{C} 13-\mathrm{H} 13 \mathrm{~B}$ & 107.6 \\
\hline $\mathrm{C} 5-\mathrm{Fe} 1-\mathrm{C} 2$ & $68.63(12)$ & $\mathrm{C} 13-\mathrm{C} 14-\mathrm{C} 15$ & $112.4(2)$ \\
\hline $\mathrm{C} 1-\mathrm{Fe} 1-\mathrm{C} 2$ & $40.93(11)$ & $\mathrm{C} 13-\mathrm{C} 14-\mathrm{H} 14 \mathrm{~A}$ & 109.1 \\
\hline $\mathrm{C} 10-\mathrm{Fe} 1-\mathrm{C} 2$ & $157.79(13)$ & $\mathrm{C} 15-\mathrm{C} 14-\mathrm{H} 14 \mathrm{~A}$ & 109.1 \\
\hline $\mathrm{C} 9-\mathrm{Fe} 1-\mathrm{C} 4$ & $106.89(12)$ & $\mathrm{C} 13-\mathrm{C} 14-\mathrm{H} 14 \mathrm{~B}$ & 109.1 \\
\hline $\mathrm{C} 5-\mathrm{Fe} 1-\mathrm{C} 4$ & $40.53(11)$ & $\mathrm{C} 15-\mathrm{C} 14-\mathrm{H} 14 \mathrm{~B}$ & 109.1 \\
\hline $\mathrm{C} 1-\mathrm{Fe} 1-\mathrm{C} 4$ & $68.75(11)$ & $\mathrm{H} 14 \mathrm{~A}-\mathrm{C} 14-\mathrm{H} 14 \mathrm{~B}$ & 107.9 \\
\hline $\mathrm{C} 10-\mathrm{Fe} 1-\mathrm{C} 4$ & $122.90(12)$ & $\mathrm{C} 16-\mathrm{C} 15-\mathrm{C} 14$ & $114.9(2)$ \\
\hline $\mathrm{C} 2-\mathrm{Fe} 1-\mathrm{C} 4$ & $68.28(12)$ & $\mathrm{C} 16-\mathrm{C} 15-\mathrm{H} 15 \mathrm{~A}$ & 108.5 \\
\hline $\mathrm{C} 9-\mathrm{Fe} 1-\mathrm{C} 3$ & $123.86(13)$ & $\mathrm{C} 14-\mathrm{C} 15-\mathrm{H} 15 \mathrm{~A}$ & 108.5 \\
\hline $\mathrm{C} 5-\mathrm{Fe} 1-\mathrm{C} 3$ & $68.59(12)$ & $\mathrm{C} 16-\mathrm{C} 15-\mathrm{H} 15 \mathrm{~B}$ & 108.5 \\
\hline $\mathrm{C} 1-\mathrm{Fe} 1-\mathrm{C} 3$ & $68.80(12)$ & $\mathrm{C} 14-\mathrm{C} 15-\mathrm{H} 15 \mathrm{~B}$ & 108.5 \\
\hline $\mathrm{C} 10-\mathrm{Fe} 1-\mathrm{C} 3$ & $159.82(13)$ & $\mathrm{H} 15 \mathrm{~A}-\mathrm{C} 15-\mathrm{H} 15 \mathrm{~B}$ & 107.5 \\
\hline $\mathrm{C} 2-\mathrm{Fe} 1-\mathrm{C} 3$ & $40.46(12)$ & $\mathrm{C} 15-\mathrm{C} 16-\mathrm{C} 17$ & $113.8(2)$ \\
\hline $\mathrm{C} 4-\mathrm{Fe} 1-\mathrm{C} 3$ & $40.79(12)$ & $\mathrm{C} 15-\mathrm{C} 16-\mathrm{H} 16 \mathrm{~A}$ & 108.8 \\
\hline $\mathrm{C} 9-\mathrm{Fe} 1-\mathrm{C} 6$ & $67.91(12)$ & $\mathrm{C} 17-\mathrm{C} 16-\mathrm{H} 16 \mathrm{~A}$ & 108.8 \\
\hline $\mathrm{C} 5-\mathrm{Fe} 1-\mathrm{C} 6$ & $124.07(12)$ & $\mathrm{C} 15-\mathrm{C} 16-\mathrm{H} 16 \mathrm{~B}$ & 108.8 \\
\hline $\mathrm{C} 1-\mathrm{Fe} 1-\mathrm{C} 6$ & $107.89(12)$ & $\mathrm{C} 17-\mathrm{C} 16-\mathrm{H} 16 \mathrm{~B}$ & 108.8 \\
\hline $\mathrm{C} 10-\mathrm{Fe} 1-\mathrm{C} 6$ & $40.69(12)$ & $\mathrm{H} 16 \mathrm{~A}-\mathrm{C} 16-\mathrm{H} 16 \mathrm{~B}$ & 107.7 \\
\hline $\mathrm{C} 2-\mathrm{Fe} 1-\mathrm{C} 6$ & $122.93(12)$ & $\mathrm{C} 18-\mathrm{C} 17-\mathrm{C} 16$ & $114.3(2)$ \\
\hline $\mathrm{C} 4-\mathrm{Fe} 1-\mathrm{C} 6$ & $160.03(13)$ & $\mathrm{C} 18-\mathrm{C} 17-\mathrm{H} 17 \mathrm{~A}$ & 108.7 \\
\hline $\mathrm{C} 3-\mathrm{Fe} 1-\mathrm{C} 6$ & $158.10(12)$ & $\mathrm{C} 16-\mathrm{C} 17-\mathrm{H} 17 \mathrm{~A}$ & 108.7 \\
\hline $\mathrm{C} 9-\mathrm{Fe} 1-\mathrm{C} 8$ & $40.16(13)$ & $\mathrm{C} 18-\mathrm{C} 17-\mathrm{H} 17 \mathrm{~B}$ & 108.7 \\
\hline $\mathrm{C} 5-\mathrm{Fe} 1-\mathrm{C} 8$ & $156.13(12)$ & $\mathrm{C} 16-\mathrm{C} 17-\mathrm{H} 17 \mathrm{~B}$ & 108.7 \\
\hline
\end{tabular}




$\begin{array}{llll}\mathrm{C} 1-\mathrm{Fe} 1-\mathrm{C} 8 & 161.73(12) & \mathrm{H} 17 \mathrm{~A}-\mathrm{C} 17-\mathrm{H} 17 \mathrm{~B} & 107.6 \\ \mathrm{C} 10-\mathrm{Fe} 1-\mathrm{C} 8 & 68.02(13) & \mathrm{C} 19-\mathrm{C} 18-\mathrm{C} 17 & 113.9(2) \\ \mathrm{C} 2-\mathrm{Fe} 1-\mathrm{C} 8 & 125.22(13) & \mathrm{C} 19-\mathrm{C} 18-\mathrm{H} 18 \mathrm{~A} & 108.8 \\ \mathrm{C} 4-\mathrm{Fe} 1-\mathrm{C} 8 & 121.53(12) & \mathrm{C} 17-\mathrm{C} 18-\mathrm{H} 18 \mathrm{~A} & 108.8 \\ \mathrm{C} 3-\mathrm{Fe} 1-\mathrm{C} 8 & 108.17(13) & \mathrm{C} 19-\mathrm{C} 18-\mathrm{H} 18 \mathrm{~B} & 108.8 \\ \mathrm{C} 6-\mathrm{Fe} 1-\mathrm{C} 8 & 67.80(13) & \mathrm{C} 17-\mathrm{C} 18-\mathrm{H} 18 \mathrm{~B} & 108.8 \\ \mathrm{C} 9-\mathrm{Fe} 1-\mathrm{C} 7 & 67.93(12) & \mathrm{H} 18 \mathrm{~A}-\mathrm{C} 18-\mathrm{H} 18 \mathrm{~B} & 107.7 \\ \mathrm{C} 5-\mathrm{Fe} 1-\mathrm{C} 7 & 161.19(12) & \mathrm{C} 20-\mathrm{C} 19-\mathrm{C} 18 & 113.7(2) \\ \mathrm{C} 1-\mathrm{Fe} 1-\mathrm{C} 7 & 124.82(12) & \mathrm{C} 20-\mathrm{C} 19-\mathrm{H} 19 \mathrm{~A} & 108.8 \\ \mathrm{C} 10-\mathrm{Fe} 1-\mathrm{C} 7 & 68.32(12) & \mathrm{C} 18-\mathrm{C} 19-\mathrm{H} 19 \mathrm{~A} & 108.8 \\ \mathrm{C} 2-\mathrm{Fe} 1-\mathrm{C} 7 & 109.00(12) & \mathrm{C} 20-\mathrm{C} 19-\mathrm{H} 19 \mathrm{~B} & 108.8 \\ \mathrm{C} 4-\mathrm{Fe} 1-\mathrm{C} 7 & 157.52(13) & \mathrm{C} 18-\mathrm{C} 19-\mathrm{H} 19 \mathrm{~B} & 108.8 \\ \mathrm{C} 3-\mathrm{Fe} 1-\mathrm{C} 7 & 122.55(12) & \mathrm{H} 19 \mathrm{~A}-\mathrm{C} 19-\mathrm{H} 19 \mathrm{~B} & 107.7 \\ \mathrm{C} 6-\mathrm{Fe} 1-\mathrm{C} 7 & 40.36(12) & \mathrm{C} 21-\mathrm{C} 20-\mathrm{C} 19 & 112.8(2) \\ \mathrm{C} 8-\mathrm{Fe} 1-\mathrm{C} 7 & 40.47(12) & \mathrm{C} 21-\mathrm{C} 20-\mathrm{H} 20 \mathrm{~A} & 109.0 \\ \mathrm{C} 7-\mathrm{C} 6-\mathrm{C} 10 & 108.3(3) & \mathrm{C} 19-\mathrm{C} 20-\mathrm{H} 20 \mathrm{~A} & 109.0 \\ \mathrm{C} 7-\mathrm{C} 6-\mathrm{Fe} 1 & 69.98(16) & \mathrm{C} 21-\mathrm{C} 20-\mathrm{H} 20 \mathrm{~B} & 109.0 \\ \mathrm{C} 10-\mathrm{C} 6-\mathrm{Fe} 1 & 69.35(17) & \mathrm{C} 19-\mathrm{C} 20-\mathrm{H} 20 \mathrm{~B} & 109.0 \\ \mathrm{C} 7-\mathrm{C} 6-\mathrm{H} 6 & 125.9 & \mathrm{H} 20 \mathrm{~A}-\mathrm{C} 20-\mathrm{H} 20 \mathrm{~B} & 107.8 \\ \mathrm{C} 10-\mathrm{C} 6-\mathrm{H} 6 & 125.9 & \mathrm{O} 21-\mathrm{C} 21-\mathrm{C} 20 & 113.8(2) \\ \mathrm{Fe} 1-\mathrm{C} 6-\mathrm{H} 6 & 126.4 & \mathrm{O} 21-\mathrm{C} 21-\mathrm{C} 11 & 148.76(17) \\ \mathrm{C} 6-\mathrm{C} 7-\mathrm{C} 8 & 107.6(3) & \mathrm{O} 21-\mathrm{C} 21-\mathrm{H} 21 \mathrm{~A} & 108.8 \\ \mathrm{C} 6-\mathrm{C} 7-\mathrm{Fe} 1 & 69.66(17) & \mathrm{C} 20-\mathrm{C} 21-\mathrm{H} 21 \mathrm{~A} & 108.8 \\ \mathrm{C} 8-\mathrm{C} 7-\mathrm{Fe} 1 & 69.73(17) & \mathrm{C} 11-\mathrm{C} 21-\mathrm{H} 21 \mathrm{~A} & 91.3 \\ \mathrm{C} 6-\mathrm{C} 7-\mathrm{H} 7 & 126.2 & \mathrm{O} 21-\mathrm{C} 21-\mathrm{H} 21 \mathrm{~B} & 108.8 \\ \mathrm{C} 8-\mathrm{C} 7-\mathrm{H} 7 & 126.2 & \mathrm{C} 20-\mathrm{C} 21-\mathrm{H} 21 \mathrm{~B} & 108.8 \\ \mathrm{Fe} 1-\mathrm{C} 7-\mathrm{H} 7 & 126.0 & \mathrm{C} 11-\mathrm{C} 21-\mathrm{H} 21 \mathrm{~B} & 107.7 \\ \mathrm{C} 9-\mathrm{C} 8-\mathrm{C} 7 & 108.0(3) & \mathrm{C} 21-\mathrm{O} 21-\mathrm{H} 21-\mathrm{H} 21 \mathrm{~B} & 109.5 \\ \mathrm{C} 9-\mathrm{C} 8-\mathrm{Fe} 1 & 69.21(18) & & \\ \mathrm{C} 7-\mathrm{C} 8-\mathrm{Fe} 1 & 69.81(17) & & \end{array}$

Hydrogen-bond geometry $\left(A,{ }^{\circ}\right)$

\begin{tabular}{lllll}
\hline$D-\mathrm{H} \cdots A$ & $D-\mathrm{H}$ & $\mathrm{H} \cdots A$ & $D \cdots A$ & $D-\mathrm{H} \cdots A$ \\
\hline $\mathrm{O} 11-\mathrm{H} 11 \cdots \mathrm{O} 21^{\mathrm{i}}$ & 0.76 & 2.06 & $2.755(3)$ & 152 \\
$\mathrm{O} 21-\mathrm{H} 21 \cdots \mathrm{O} 11^{\mathrm{ii}}$ & 0.83 & 1.90 & $2.726(3)$ & 175 \\
$\mathrm{C} 6-\mathrm{H} 6 \cdots \mathrm{O} 21^{\mathrm{i}}$ & 0.95 & 2.60 & $3.380(4)$ & 140 \\
\hline
\end{tabular}

Symmetry codes: (i) $-x+1 / 2, y-1 / 2,-z+3 / 2$; (ii) $-x+1 / 2,-y+3 / 2,-z+2$. 